Exclusion criteria: individual case studies, conference abstracts and opinion pieces. No date or language restrictions.

Search terms included: 'pulmonary rehabilitation' AND 'referral' OR 'uptake' applied to MEDLINE, EMBASE, CINAHL, PsychINFO, ASSIA, BNI, Web of Science and Cochrane Library from inception to June 2016 supplemented by review of reference lists and citation search. Titles, abstracts and full papers were reviewed independently, quality appraised (using Cochrane Collaboration's tool for RCTs and ROBINS-I, AMSTAR) and entered into summary tables. The protocol was registered (PROSPERO) and reported according to PRISMA guidelines.

Results We screened 3217 references, from which 7 papers including 6345 patients and 22 clinicians met inclusion criteria. Most studies $(\mathrm{n}=5)$ were UK based.

Designs, interventions and scope of studies were diverse with interventions often part of multifaceted evidence based management of COPD. Examples included computer based prompts at practice nurse review, patient information, financial incentives. Most studies $(n=5)$ reported improvements in referral or uptake of PR (range 0\% - 25\% increase), however most had methodological limitations with risk of bias. Due to heterogeneity, studies were not considered combinable and meta-analysis was inappropriate.

Conclusions There is limited evidence for the efficacy of interventions to increase referral and uptake of PR. Existing studies are diverse and further testing using robust methods in various populations and settings is required to optimise access to PR.

\section{P213 POSITIVE DRIVERS AND POTENTIAL BARRIERS TO IMPLEMENTATION OF HOSPITAL AT HOME SELECTED BY LOW RISK DECAF SCORE}

${ }^{1} \mathrm{~L}$ Dismore, ${ }^{1} \mathrm{C}$ Echevarria, ${ }^{2} \mathrm{~A}$ Van-Wersch, ${ }^{3} \mathrm{AJ}$ Simpson, ${ }^{3} \mathrm{GJ}$ Gibson, ${ }^{1} \mathrm{SC}$ Bourke. ${ }^{1}$ North Tyneside General Hospital, North Shields, UK; ${ }^{2}$ Teesside University, Teesside, UK; ${ }^{3}$ Newcastle University, Newcastle upon Tyne, UK

\subsection{6/thoraxjnl-2016-209333.356}

Background Despite endorsement in guidelines, many hospitals do not offer hospital at home (HAH) for COPD exacerbation (AECOPD), partly reflecting the previous lack of a robust prognostic score to guide selection. The DECAF score addresses this concern, and should be routinely scored on admission. ${ }^{1}$ In a RCT we have shown that HAH selected by DECAF score $0-1$ is safe and effective. Up to $50 \%$ of admitted patients are suitable. Our population included patients with higher medical dependency than earlier trials and HAH was supported by $24 / 7$ specialist oncall. In an embedded qualitative study, we identified positive drivers for, and potential barriers to, use of $\mathrm{HAH}$ to inform service implementation.

Methods Patients, carers, clinicians and managers were purposely selected to ensure diversity. Semi-structured interviews were conducted and Thematic-Construct Analysis employed. ${ }^{2}$

Results 44 patients (HAH/inpatient care/declined randomisation), 15 carers, 14 consultants, 11 specialist nurses and 4 managers were interviewed. 'Positive drivers' were divided into two subconstructs: 'Feeling more at ease and comfortable in own home environment'; and 'Feeling safe, reassured and appreciated through continuity of hospital care'. Positive influences on independence, perceived rate of recovery, sleep quality, mood and contact with friends and family were noted. At 14 days post-presentation, $90 \%$ of patients stated they would prefer HAH over inpatient care for subsequent exacerbations of similar severity.
Counter-intuitively, carers reported greater convenience rather than increased burden.

'Potential Barriers' were grouped into two sub-constructs: 'Personal preferences'; and 'Resistance to change'. Some patients highlighted fear of being alone at night and dislike of strangers visiting their home; nurses cited higher workload and greater responsibility (with experience, viewed positively); whilst operational concerns included keeping medical records in a patient's home and inability to capture activity within current payment systems.

Conclusions HAH selected by DECAF allows the inclusion of more patients than existing models, and is preferred to inpatient care by most patients and their families. During the trial few barriers to implementation were identified, and were effectively overcome. Hospitals planning to implement $\mathrm{HAH}$ selected by DECAF should pre-emptively address these issues.

\section{REFERENCES}

1 National COPD Audit Report, 2015

2 Dismore. J Health psychol, 2016.

\section{P214 THE PREVALENCE OF RESPIRATORY SYMPTOMS AND LUNG DISEASE IN A SOUTH LONDON "LUNG HEALTH IN ADDICTIONS" SERVICE}

${ }^{1} \mathrm{CJ}$ Jolley, ${ }^{2} \mathrm{~A}$ Healey, ${ }^{3} \mathrm{R}$ Cole, ${ }^{3} \mathrm{R}$ Baker-Wilding, ${ }^{4} \mathrm{~B}$ Tas, ${ }^{5} \mathrm{~J}$ Moxham, ${ }^{4} \mathrm{~J}$ Strang, ${ }^{3} \mathrm{M}$ Kelleher. ${ }^{1}$ Centre of Human and Aerospace Physiological Sciences, King's College London, London, UK; ${ }^{2}$ CLAHRC South London/King's Improvement Science, King's College London, London, UK; ' ${ }^{2}$ Lorraine Hewitt House, Lambeth Addictions Consortium, South London and Maudsley NHS Foundation Trust, London, UK; ${ }^{4}$ National Addiction Centre, Institute of Psychiatry, Psychology and Neuroscience (IoPPN) at King's College London, London, UK; ${ }^{5}$ Division of Asthma, Allergy and Lung Biology, King's College London, London, UK

\subsection{6/thoraxjnl-2016-209333.357}

Introduction and objectives Patients undergoing treatment for heroin addiction die on average 15 years earlier than the general population (Smyth et al., 2006). Opioid-related deaths in England and Wales have increased by two-thirds since 2012 (Office for National Statistics 2015). Although smoking-related respiratory disease contributes to the excess mortality in drug and alcohol addiction, screening for lung disease is not routinely offered in community drug and alcohol treatment services (CDATs). We have established a "Lung Health Clinic" in our local CDAT (Brixton, Lambeth, SE London). Through this clinic we aimed to document the respiratory symptom burden, and COPD prevalence, in addicts accessing our local CDAT.

Methods Assessments: handheld spirometry (FEV $1, \mathrm{FVC}, \mathrm{FEV}_{1} /$ FVC ratio) and pulse oximetry $\left(\mathrm{SpO}_{2} \%\right)$. We recorded breathlessness (mMRC Dyspnoea Scale), chronic cough and cigarette smoking status.

Results 112 patients (88 male, mean(SD) age 46 (9) years) attended Jan-Dec 2015. Current/previous addictions included: smoked heroin $66.3 \%$, intravenous heroin $32.5 \%$, crack cocaine $68.2 \%$, cannabis $60.0 \%$. $81.3 \%$ were current cigarette smokers. Only $41 \%$ had accessed smoking cessation services despite these being offered in-house.

$61.5 \%$ reported chronic cough. 20\% reported significant breathlessness (mMRC Dyspnoea $\geq 2$ ). $5 \%$ had a pre-existing COPD diagnosis, whereas spirometry [Figure 1] and clinical history were consistent with $\mathrm{COPD}$ in $36.4 \% . \mathrm{SpO}_{2} \leq 95 \%$ in $12.8 \%$.

Conclusions There is a significant unmet burden of undiagnosed chronic lung disease, and respiratory symptoms, in our local 